\title{
Effect of Annealing on Optical Properties of Zinc Oxide Thin Films Prepared by Homemade Spin Coater
}

\author{
Deependra Das Mulmi, Agni Dhakal and Buddha Ram Shah \\ Nepal Academy of Science and Technology, Lalitpur, Nepal \\ e-mail:dmulmi@gmail.com
}

\begin{abstract}
Zinc oxide $(\mathrm{ZnO})$ thin films were deposited on the ordinary glass substrates by spin coating method. The precursor solution was prepared by mixing zinc acetate dihydrate in appropriate proportions with ethanol and diethanolamine (DEA). The obtained thin films were dried at $200^{\circ} \mathrm{C}$ for 15 minutes in hot air oven. Crystalline $\mathrm{ZnO}$ thin films were achieved following annealing process at temperatures $300^{\circ}, 400^{\circ}$ and $500^{\circ} \mathrm{C}$ for 2 hours. Thin films as- prepared were studied by X-ray diffraction and UV-visible spectroscopy. The films were transparent from near ultraviolet to infrared region. Optical band gap energy of $\mathrm{ZnO}$ was obtained $3.22 \mathrm{eV}$ at $300^{\circ} \mathrm{C}$. On annealing at $400^{\circ}$ and $500^{\circ} \mathrm{C}$, band gap energy was shifted at $3.14 \mathrm{eV}$ and $3.05 \mathrm{eV}$ respectively.
\end{abstract}

Key words: sol-gel method, zinc oxide, absorption spectra, annealing, band gap energy

\section{Introduction}

Semiconducting devices are mostly based on advancement of thin film technology. Thin film is a two dimensional material deposited by either atom-by-atom or molecule-by-molecule condensation method. The thin film technology is deposition of molecules. On the other hand, the thick film is deposition of particles. Oxide semiconductors have drawn much attention because of their wide range of potential device applications, from transistors to optical detection and emission. In this context, $\mathrm{ZnO}$ is of a particular interest because of its wide direct band gap energy of $3.37 \mathrm{eV}$ and a large binding energy of $60 \mathrm{meV}$ (Liang \& Yoffe 1968) that makes it one of the best candidates for transparent field effect transistors, gas sensors and solar cells (Djurišiæ et al. 2010). Moreover, $\mathrm{ZnO}$ is a promising material for short wavelength optoelectronic devices, especially for ultraviolet light-emitting diodes (UV LEDs) and laser diodes (LDs). This exciton binding energy $(60 \mathrm{meV})$ is much larger than the room temperature thermal energy (26 meV), suggesting that the electron-hole pairs are stable even at room temperature. Therefore, efficient UV LEDs and LDs operating at room temperature can be expected, provided that high-quality p-type $\mathrm{ZnO}$ is available. A great effort has been brought out to improve the electrical and optical properties of $\mathrm{ZnO}$ films through a diversity of material synthesis techniques, substrates, doping and processing. Up to now, several methods have been used to fabricate high quality $\mathrm{ZnO}$ thin films such as pulsed laser deposition (Craciun et al. 1994), molecular beam epitaxy (Heo et al. 2006), metal organic chemical vapor deposition (Tan et al. 2005), direct current and radio-frequency magnetron sputtering (Cho 2009), solgel (Ghodsi et al. 2010), and spray pyrolysis (Ayouchi et al. 2003 and Godbole et al. 2011). Among the growth methods, the sol-gel method shows many advantages over other deposition techniques, such as its simplicity, ease of control of the chemical composition of the thin films, and low equipment cost. In addition, it deposits films with large areas and good thickness uniformity. However, the crystalline quality of the $\mathrm{ZnO}$ prepared by sol-gel process might be inferior to other methods (Borgogno et al. 1982). This effect is caused by the low temperature annealing performed in the sol-gel process that affects the structural and optical characteristics of the thin films. The present study deals with preparation of $\mathrm{ZnO}$ thin films by Sol-gel method using homemade spin coater. Also this work reports structural and optical characterization of $\mathrm{ZnO}$ thin films by varying temperature. 


\section{Methodology}

The precursor solution can be deposited on a suitable substrate by various coating techniques. The mainly used techniques for thin film preparation from the Sol-gel route are either spin coating or dip coating. Spin coating allows the preparation of thin films on flat substrates. An adequate amount of solution is dropped on a rotating substrate. The solution spreads on the surface and forms a film. In second step, the speed of rotation increases and not required rest of the solution will be removed by centrifugal forces. In this step, the volatile solvent evaporates and a dry, thin, metal organic film is formed. The thickness of the film depends on the viscosity, the concentration of the solution and on the angular speed of the spin coater. With increasing angular speed the film thickness decreases. Zinc oxide thin films were deposited on glass substrates by spin coating method. As a starting material, $0.5 \mathrm{M}$ of zinc acetate dihydrate $\left[\left(\mathrm{CH}_{3} \mathrm{COO}\right)_{2} \mathrm{Zn} .2 \mathrm{H}_{2} \mathrm{O}\right]$ was used. The precursor solution was prepared by mixing zinc acetate dihydrate in appropriate proportions with ethanol and diethanolamine (DEA). Here ethanol and DEA were used as solvent and stabilizer respectively. The glass substrate (often used as a slide in microscope) was first cut into two equal pieces using a diamond glass cutter and washed by detergent, and cleaned in distilled water and acetone, and then dried in oven at $200^{\circ} \mathrm{C}$ for 2 hours. The coating solution was dropped using a syringe as shown in Fig. 1(a) onto the glass substrate. Then the substrate with the solution was rotated at $3000 \mathrm{rpm}$ for $30 \mathrm{sec}$ by using homemade spin coater as shown in Fig. 2(b) and 2(c). After depositing by spin coating, the film was dried at $200^{\circ} \mathrm{C}$ for $15 \mathrm{mins}$ in a hot air oven in order to evaporate the solvent and remove organic residuals. The procedures from coating to drying were repeated six times. The film was then inserted into a muffle furnace and annealed in air at $300^{\circ}$, $400^{\circ}$ and $500^{\circ} \mathrm{C}$ for $2 \mathrm{hrs}$. The process of thin film growing could be well understood from the flow chart as shown in Fig. 2.

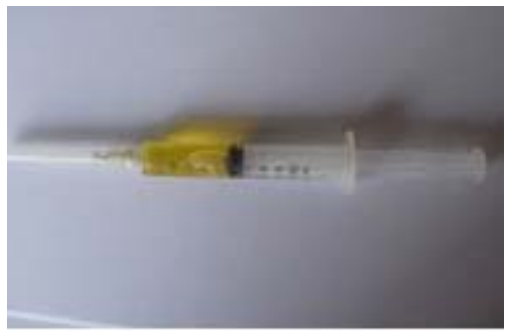

Fig. 1. Steps involved in homemade spin coating process.

(a) a medical syringe is used to drop the coating solution on the glass substrate

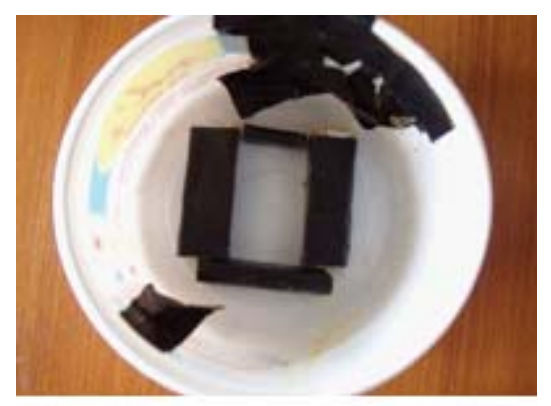

(b) a sample holder made from plastic ice cream box

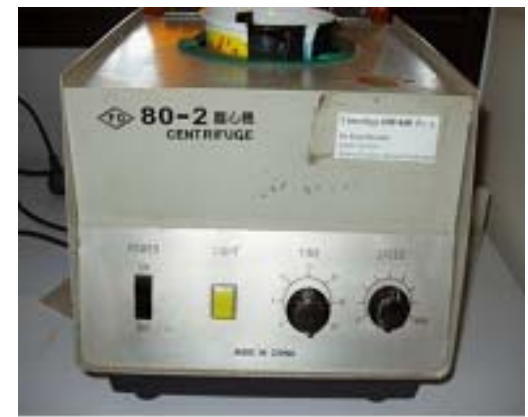

(c) a centrifuge machine is modified as a homemade spin coater

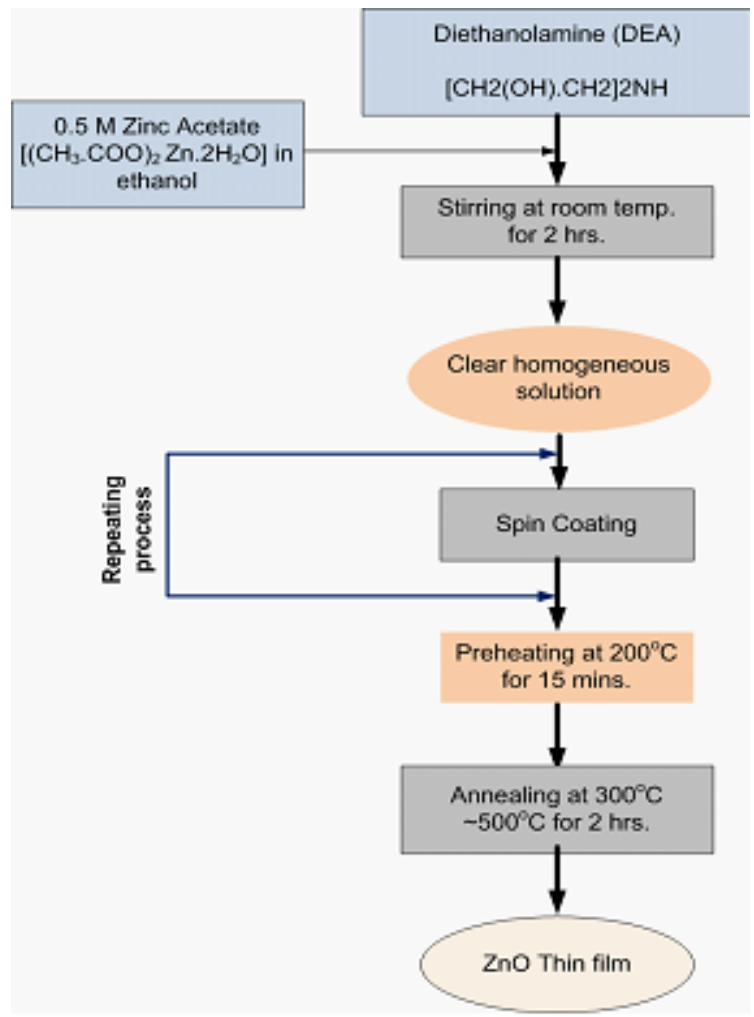

Fig. 2. Flow chart showing the procedure of $\mathrm{ZnO}$ thin films preparation 


\section{Results and Discussion}

\section{Structure characterization}

$\mathrm{X}$-ray diffraction (XRD) patterns of the sample were taken with a D2 phaser Bruker X-ray diffractometer at room temperature using $\mathrm{CuK} \alpha$ radiation wavelength of $\lambda=1.542 \AA$. The peak position and intensity were obtained between 0 and $80^{\circ}$. Fig. 3 shows the powder $\mathrm{X}$-ray diffraction pattern of the as-grown $\mathrm{ZnO}$ thin films. The diffraction lines are consistent with the values reported in the database of $\mathrm{ZnO}$ (JCPDS card no PDF 89-1397) providing clear evidence for the formation of hexagonal Wurtzite-type structure of the synthesized $\mathrm{ZnO}$. The as-grown $\mathrm{ZnO}$ thin films were of pure sample and no diffraction peaks from any other impurities were detected. All the diffraction peaks are rather sharp which indicate that the $\mathrm{ZnO}$ sample has high degree of crystallanity. From the XRD data, the crystallite size $(D)$ of the as-grown $\mathrm{ZnO}$ thin films was calculated to $39.54 \mathrm{~nm}$, using the Debye-Scherrer equation (Khan et al. 2011),

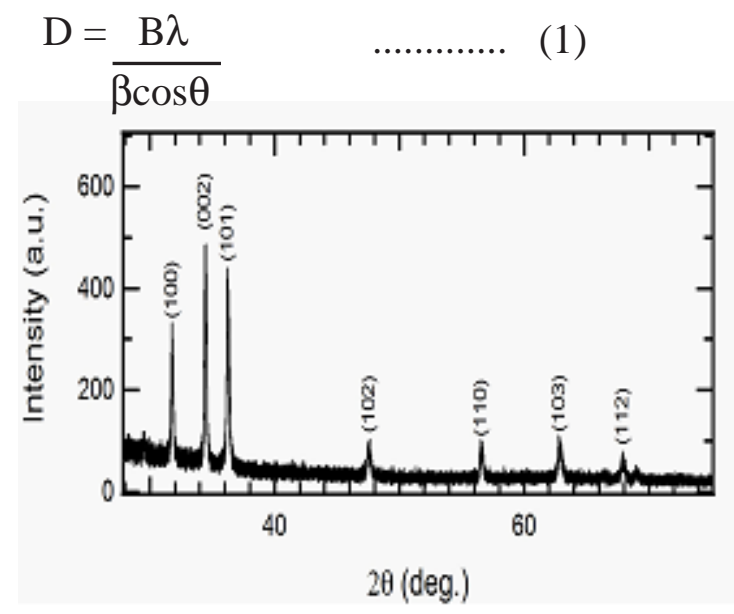

Fig 3. XRD patterns of $\mathrm{ZnO}$ thin film on glass substrate at room temperature

In this equation, $D$ is the crystallite size (nm) of the phase under investigation, B is the Scherrer constant (0.9), $\lambda$ is the wavelength of $\mathrm{X}$-ray of $\mathrm{Cu} \mathrm{K} \alpha=0.154$ $\mathrm{nm}, \beta$ is the full width half maximum (FWHM) of (002) reflection peak as shown in Fig. 4 and $\theta$ is the Bragg's angle. The calculated crystallite size in the present study was very close to that reported by Kumar et al. (2011). Peaks corresponding to the (100), (002), (101), (102), (110), (103) and (112) planes of hexagonal $\mathrm{ZnO}$ were observed. No other peaks were observed, suggesting that only single-phase $\mathrm{ZnO}$ was formed.

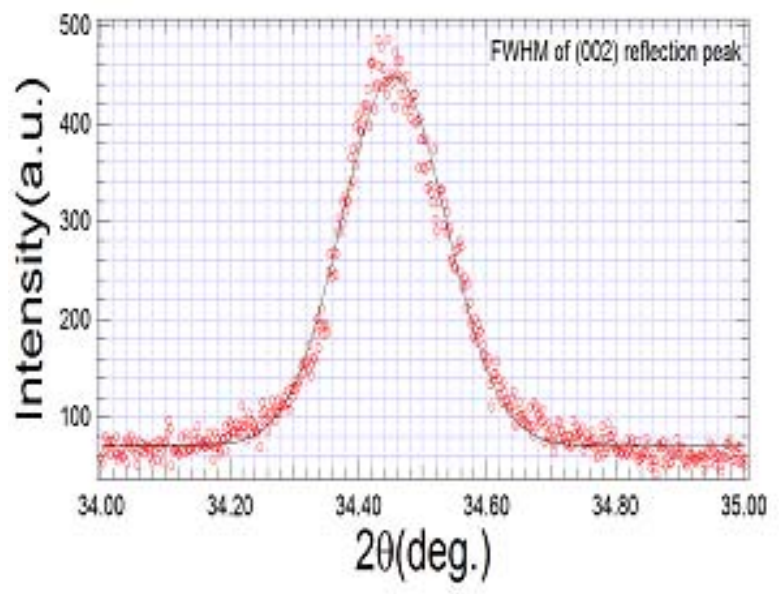

Fig 4. Full width at half maximum of (002) reflection peak used for the calculation of the crystallite size. The circles and line represent the experimental values and curve fitting respectively

\section{Optical properties of $\mathrm{ZnO}$}

Band gaps are one of the most important properties of modern semiconductors. With applications in photovoltaic, visual display and lighting sources, the band gap of a semiconductor is an essential property for designing and discovering new applications for semiconductors. The most common method of determining the band gap of a semiconductor was by optical absorption or transmission measurements. For measuring the band gap energy of a material, the optical absorption method was applied using USB 2000 spectrophotometer of Ocean Optics. In this measurement, photons of selected wavelength fell on the sample and the transmitted photons were measured. This experiment could calculate an exact value of the band gap energy because photons with energies higher than the band gap energy were absorbed and photons with energies lower than the band gap were transmitted. The experimental assembly provided electromagnetic radiation in the wide range of $200 \mathrm{~nm}$ to $1100 \mathrm{~nm}$. In the long wavelength limit, radiation could excite free charge carriers to oscillate causing reflection. In short wavelength limit, radiation had enough energy to interact with inner shell electrons. The room temperature optical absorption of $\mathrm{ZnO}$ thin films annealed at different temperatures has been shown in Fig.5. The films showed a sharp absorption around $400 \mathrm{~nm}$ and almost transparent in the visible range. 


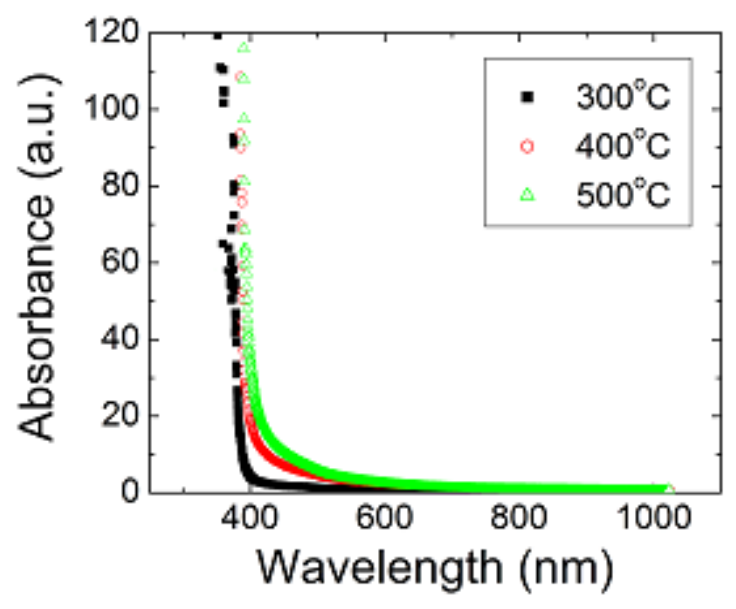

Fig. 5. The room temperature optical absorption of $\mathrm{ZnO}$ thin films annealed at different temperatures

\section{Band gap energy of $\mathrm{ZnO}$}

Determination of band gap energy $\left(E_{\mathrm{g}}\right)$ was often necessary to develop the electronic band structure of a thin film material. Absorption coefficient $a ́$ was related to the energy $h i$ of incident photons by the relation called Tauc's plot method (Ding et al. 2010):

$$
\alpha h v=B\left(h v-E_{g}\right)^{p}
$$

where $B$ was a constant and $p$ was an index that characterized the optical absorption process and was theoretically equal to $1 / 2,2,3 / 2$ or 3 for direct allowed, indirect allowed, direct forbidden and indirect forbidden transitions, respectively. In the present study of $\mathrm{ZnO}$, the value of $p$ of Eq. (2) was taken as $1 / 2$ (Gümü et al. 2006). Absorption coefficient, á, was mainly influenced by two factors: (i) scattering losses and (ii) fundamental absorption. At shorter wavelengths close to the optical band gap, the influence of fundamental absorption on $a$ was more prominent than to scattering losses and $a$ might be obtained by (Kumar et al. 2011):

$\alpha=\left[\frac{1}{d} \ln \left(\frac{1}{T}\right)\right] \ldots$

The band gap variation of $\mathrm{ZnO}$ with annealing temperature has been shown in Fig. 6. The optical band gap $(\mathrm{Eg})$ was obtained by extrapolating the linear part of the Tauc's plot to intercept the energy axis at $(\text { áhí })^{2}=0$. The optical band gap energies versus temperature as determined from the obtained optical spectra have been shown in Fig.7. The optical band gap for the $\mathrm{ZnO}$ film grown at $300^{\circ} \mathrm{C}$ was $3.22 \mathrm{eV}$. As the growth temperature was increased to $400^{\circ}$ and $500^{\circ} \mathrm{C}$, the optical band gap shifted to 3.14 and 3.05 $\mathrm{eV}$ respectively. It was found that there was a narrowing of the band gap of about $170 \mathrm{meV}$ between the two samples deposited at $300^{\circ}$ and $500^{\circ} \mathrm{C}$. These results were consistent with those observed for the mechanical assisted thermal decomposition method (Zhang et al. 2014).

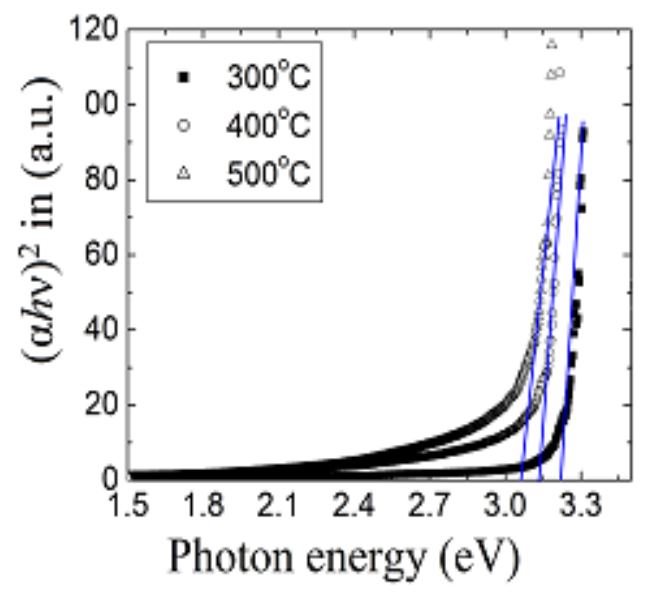

Fig.6. Graph between (áhí) ${ }^{2}$ and photon energy

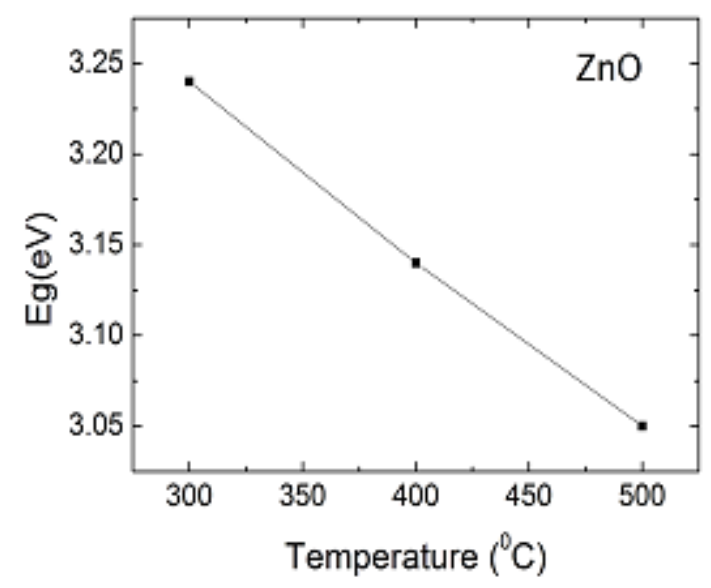

Fig.7. Band gap energy of $\mathrm{ZnO}$ annealed at different temperatures

The band gap energy of the $\mathrm{ZnO}$ thin films decreased with temperature. Compared with the band gap energy of $\mathrm{ZnO}$ single crystal $(3.3 \mathrm{eV})$, the band gap energy of the $\mathrm{ZnO}$ thin film $(3.22 \mathrm{eV})$ was somewhat smaller. The small variation of the band gap energy might be originated from the defects in $\mathrm{ZnO}$ thin films. 


\section{Acknowledgements}

We would like to thank Prof. Dr. Shanker Prasad Shrestha, Patan Multiple Campus for the optical absorption measurement. We are also grateful to Dr. Suresh Kumar Dhungel and Mr. Hari Ram Shrestha for the XRD measurement.

\section{References}

Ayouchi, R., D. Leinen, F. Martin and J. R. Ramos-Barrado. 2003. Growth of pure $\mathrm{ZnO}$ thin films prepared by chemical spray pyrolysis on silicon. J. Cryst. Growth 247: 497-504.

Borgogno, J.P., B. Lazarides and E.Pelletier.1982.Automatic determination of the optical constants of inhomogeneous thin films. Appl. Opt. 21: 4020-4029.

Cho, S., 2009.Effects of growth temperature on the properties of $\mathrm{ZnO}$ thin films grown by radio-frequency magnetron sputtering, Transaction on Electrical and Electronic Materials 10:185-188.

Craciun, V., J. elders, J. G. E. Gardeniers and I. W. Boyd.1994. Characteristics of high quality $\mathrm{ZnO}$ thin films deposited by pulsed laser deposition, Appl. Phys.Lett.65: 2963-2965.

Ding, R., C. Xu, B. Gu, Z. Shi, H. Wang, L. Ba and Z. Xiao 2010.Effects of $\mathrm{Mg}$ incorporation on microstructure and optical properties of $\mathrm{ZnO}$ thin films prepared by sol-gel method. J. Mater. Sci. Technol. 26(7):601-604.

Djurišiæ, A.B., A.M. C. Ng and X.Y. Chen. 2010. ZnO nanostructures for optoelectronics: Material properties and device applications. Progress in Quantum Electronics 34:191-159.
Ghodsi, F.E. and H.Absalan.2010.Effects of growth temperature on the properties of $\mathrm{ZnO}$ thin films grown by radio-frequency magnetron sputtering. ActaPhysicaPolonica A.118: 659-664.

Godbole, B., N. Badera, S. Shrivastava, D. Jain and V. Ganesan. 2011. Growth mechanism of $\mathrm{ZnO}$ films deposited by spray pyrolysis technique. Mat. Sc. and Appl.2: 643-648.

Gümü, C., O.M. Ozkendir, H. Kavak and Y. Ufuktepe.2006.Structural and optical properties of zinc oxide thin films prepared by spray pyrolysis method. J. Optoelectronics and Advanced Materials 8:299-303.

Heo, Y.W., K.Ip, S.J. Pearton, D.P. Norton and J.D. Budai.2006. Growth of $\mathrm{ZnO}$ thin films on c-plane $\mathrm{Al}_{2} \mathrm{O}_{3}$ by molecular beam epitaxy using ozone as an oxygen source. Appl. Surf. Sc.252:7442-7448.

Khan, Z.R., M.S. Khan, M. Zulfequar and M.S. Khan. 2011. Optical and structural properties of $\mathrm{ZnO}$ thin films fabricated by sol-gel method. Mat. Sc. and Appl. 2: 340-345.

Kumar, V., R.G. Singh, L.P. Purohit and R.M. Mehra. 2011.Structural, transport and optical properties of Boron-doped zinc oxide nanocrystalline. J. Mater. Sci. Technol. 27(6):481-488.

Liang, W.Y. and A.D. Yoffe.1968.Transmission spectra of ZnO single crystals. Phys. Rev. Lett. 20: 59-62.

Zhang,X., J. Qin, Y.Xue1, P. Yu, B. Zhang, L. Wang and R. Liu. 2014. Effect of aspect ratio and surface defectson the photocatalytic activity of ZnOnanorods. Scientific Reports.4:45961- 45968. URL http:// www.nature.com/srep/2014/140404/srep04596/pdf/ srep04596.pdf (DOI: 10.1038/srep04596) 
Nepal Journal of Science and Technology Vol. 15, No.2 (2014) 111-116 